\title{
Helina fratercula (Zetterstedt, 1845) (Diptera: Muscidae) newly recorded from China, with a redescription of male
}

\author{
Ming Zhang, Mingfu Wang \& Dong Zhang*
}

\begin{abstract}
Zhang, M., Wang, M. F. \& Zhang, D. 2015: Helina fratercula (Zetterstedt, 1845) (Diptera: Muscidae) newly recorded from China, with a redescription of male. Entomol. Fennica 26: 117-121.
\end{abstract}

Helina fratercula (Zetterstedt, 1845), so far known only from Central Europe, is newly recorded from China. The species is redescribed in detail morphological characters. The characteristic photos and the illustrations of male terminalia based on the specimens from Xinjiang are provided, and also incorporated into the existing key of Helina males of China.

M. Zhang \& D. Zhang, School of Nature Conservation, Beijing Forestry University, Beijing 100083, P. R. China; *Corresponding author's e-mail: ernest 8445 @163.com

M. F. Wang, Institute of Entomology, Shenyang Normal University, Shenyang 110034, P. R. China

Received 4 December 2014, accepted 19 February 2015

\section{Introduction}

Helina Robineau-Desvoidy, 1830 is the second largest genus of Muscidae, comprising nearly 600 described species and more new species awaiting description (Pont 1980, 1986, Carvalho et al. 2005, Wang et al. 2008, Wang 2013). Faunal diversity of Helina is particularly impressive in the Chinese fauna, where recent inventories have revealed up to $40 \%$ of the known world species (Xue \& Chao 1998, Xue et al. 2005, Wang et al. 2008). It is an intimidating task to identify the species correctly due to Helina including so many species and similar morphological characters. Morphology as a source of information still remains to be explored in Helina systematics, because a part of the species has not been adequately described previously. Consequently, detailed morphological information for this genus is very important. Such character sets are a rich potential source not only for identifying species but for further phylogenetic studies.
A faunal survey of Muscidae was conducted in the mountainous region of northern part of Xinjiang Uygur Autonomous Region, Northwestern China in the summer of 2014. The Muscidae fauna in this area had not been previously investigated in detail. After having checked extensively specimens in the collections of the Museum of Beijing Forestry University (MBFU), we confirm the presence of Helina fratercula (Zetterstedt, 1845) in China for the first time and provide a redescription, characteristic photos and illustrations of male terminalia.

\section{Material and methods}

The specimens of the newly recorded species examined in this study were collected in June of 2014 by sweeping from brushwood in the mountainous region of Xinjiang. They were killed with ethylacetate and pinned while the specimens were still fresh, then deposited in the Museum of 


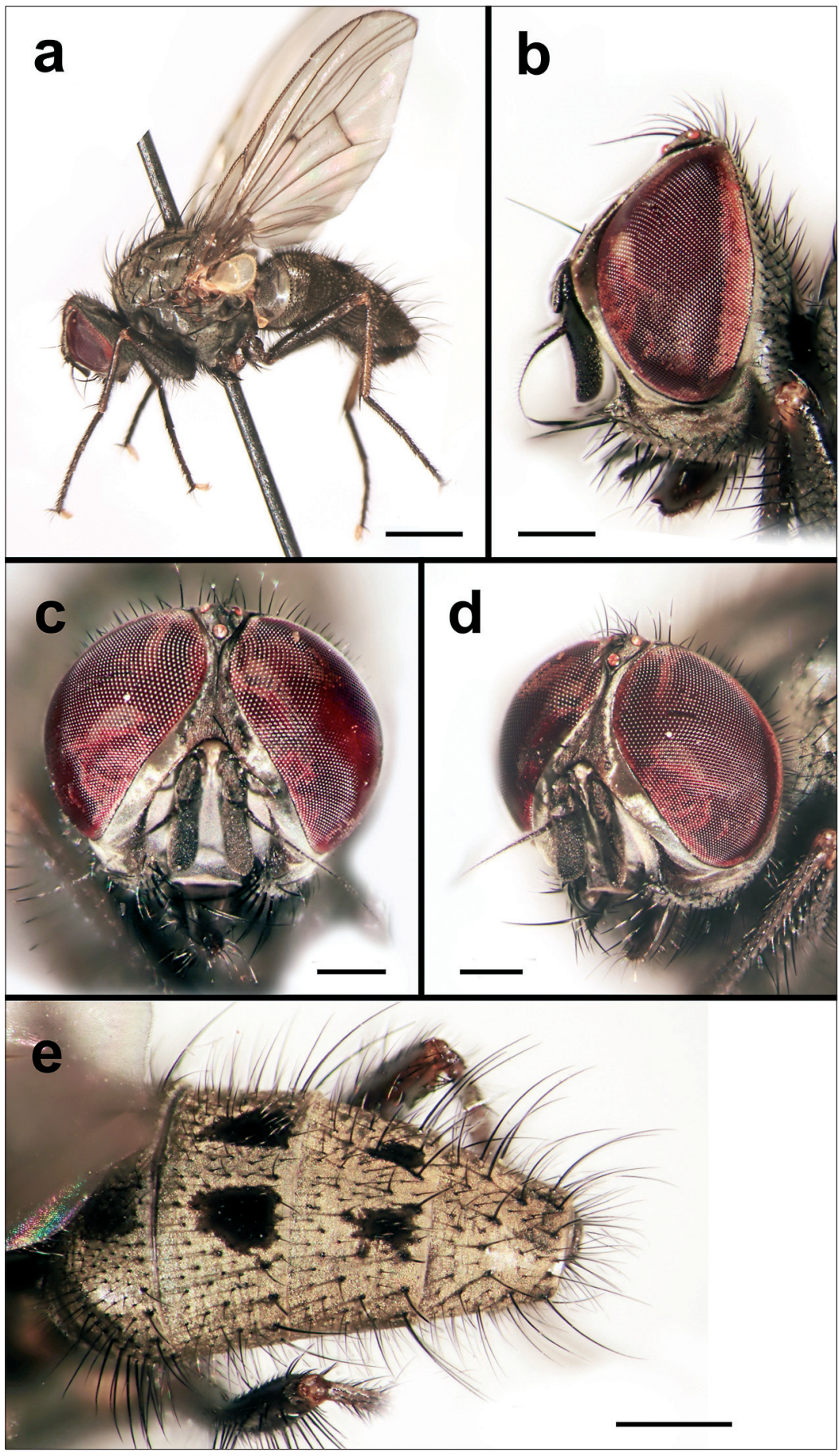

Fig. 1. Photographs of male Helina fratercula (Zetterstedt, 1845). - a. Body, lateral view, scale bar $2.0 \mathrm{~mm}$. - b. Head, lateral view, scale bar $0.5 \mathrm{~mm}$. - c. Head, anterior view, scale bar 0.5 $\mathrm{mm}$. - d. Head, anterolateral view, scale bar $0.5 \mathrm{~mm}$. - e. Abdomen, laterodorsal view, scale bar $1.0 \mathrm{~mm}$.

Beijing Forestry University (MBFU), Beijing, China. The male terminalia was detached from the body, cleared in hot $10 \% \mathrm{KOH}$ solution (at approximately $100{ }^{\circ} \mathrm{C}$ ) for about five minutes, then placed in glycerine. The specimens were ex- amined, illustrated and measured using a stereoscopic microscope Olympus SZX16.

We used the terminology of adult fly structure of McAlpine (1981) with the exception of "postpedicel" for the first antennal flagellomere, 
which followed Stuckenberg (1999). The abbreviations used in the description are as follows: $\mathrm{acr}$ $=$ acrostichal seta, $a d=$ anterodorsal seta, $a v=$ anteroventral seta, $d=$ dorsal seta, $d c=$ dorsocentral seta, $i a=$ intra-alar seta, $p=$ posterior seta, $p d=$ posterodorsal seta, $p r a=$ prealar seta, and $p v$ $=$ posteroventral seta

\section{Taxonomy}

Helina fratercula (Zetterstedt, 1845) (Figs 1-3)

Material examined. One male: China, Xinjiang, Altay, Xiaodonggou, $47^{\circ} 58^{\prime} \mathrm{N}, 88^{\circ} 12^{\prime} \mathrm{E}$, alt. 1,100 m, 2.VI.2014, Dong Zhang and Ming Zhang leg.; one male: China, Xinjiang, Altay, Buerjin, Hanasi, $48^{\circ} 10^{\prime} \mathrm{N}, 87^{\circ} 30^{\prime} \mathrm{E}$, alt. 1,430 m, 4.VI.2014, Dong Zhang leg.

Redescription of male. Body length about $9.00 \mathrm{~mm}$.

Head. Eyes with sparse and short hairs, facets a little expanded on anterior margin in upper part; frons slightly narrower than distance between outer margins of posterior ocelli, and about 1.20 times as wide as postpedicel, frontal vitta black, at narrowest point as wide as fronto-orbital plate, 5 frontal setae, upper orbital setae absent, frontoorbital plate and parafacial with silvery pollinosity, parafacial about 1.20 times as wide as postpedicel; antenna black, postpedicel about 2.50 times as long as wide, arista with short plumes, longest hair shorter than $1 / 2$ of width of antennal flagellomere; lunule yellow; epistoma not projecting, vibrissal angle behind frontal angle in profile; gena with grey pollinosity, gena and metacephalon with black hairs; proboscis short, labella large, palpus black, slender and long.

Thorax. Ground-colour black with dense grey pruinescence, scutum with four dark vittae in posterior view, median pair not reaching scutoscutellar suture; chaetotaxy: acr $0+1, d c 2+3$, ia $1+2$, pra 2 (with 1 hair-like); ventral and lateral margins of scutellum bare; basisternum, proepisternum, anepimeron, katepimeron and meron bare; katepisternal setae 1:2, with strong setae on lower margin (Fig. 2d); anterior spiracle yellowish, posterior one brown. Wings. Translucent, tegula and basicosta brownish-yellow, costal

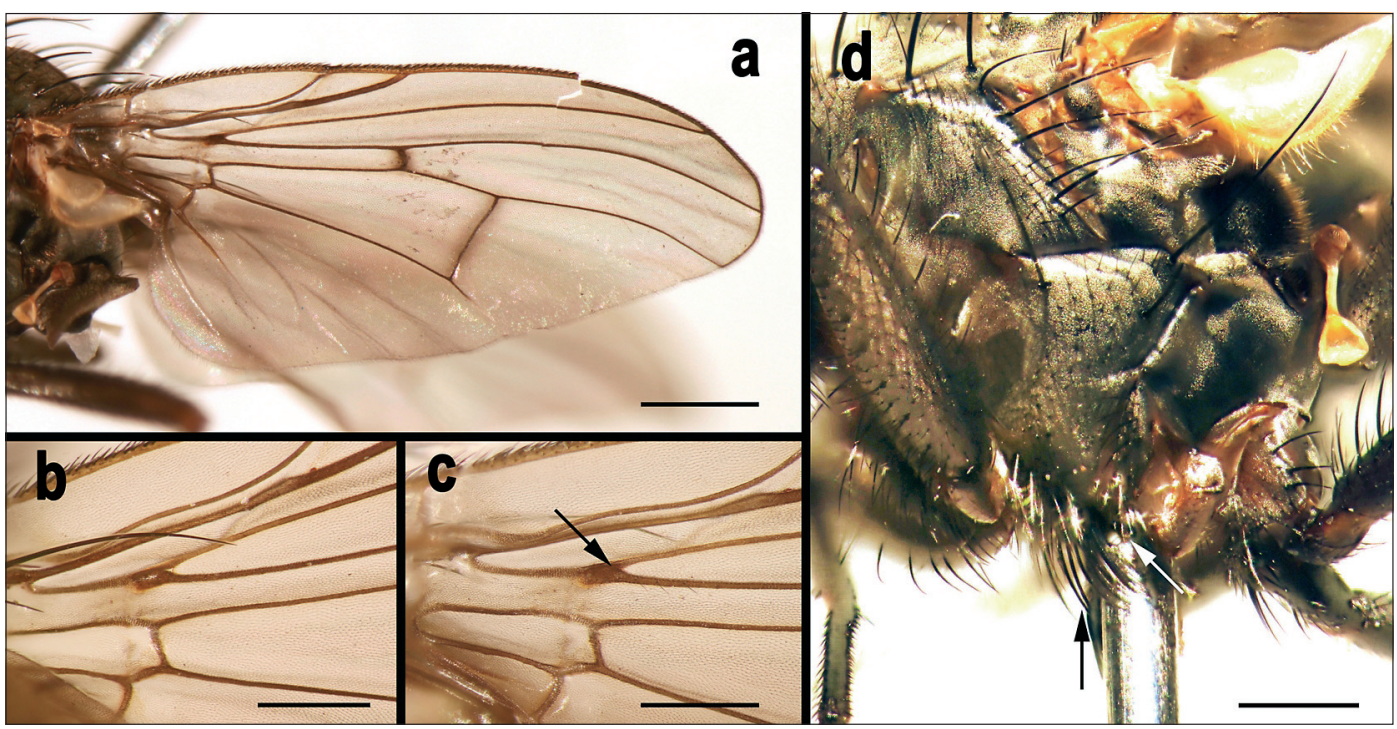

Fig. 2. Photographs of male Helina fratercula (Zetterstedt, 1845). - a. Left wing, ventral view, scale bar $1.0 \mathrm{~mm}$. - b. Left wing, dorsal view, node of Rs, scale bar $0.5 \mathrm{~mm}$. - c. Left wing, ventral view, node of Rs, arrow showing the setae on the ventral surface of node Rs, scale bar $0.5 \mathrm{~mm}$. - d. Mid thorax, lateral view, showing the katepisternal setae 1:2 and strong setae on lower margin (black arrow), as well as mid coxa with strong setae on lower and inner margins (white arrow); scale bar $0.5 \mathrm{~mm}$. 

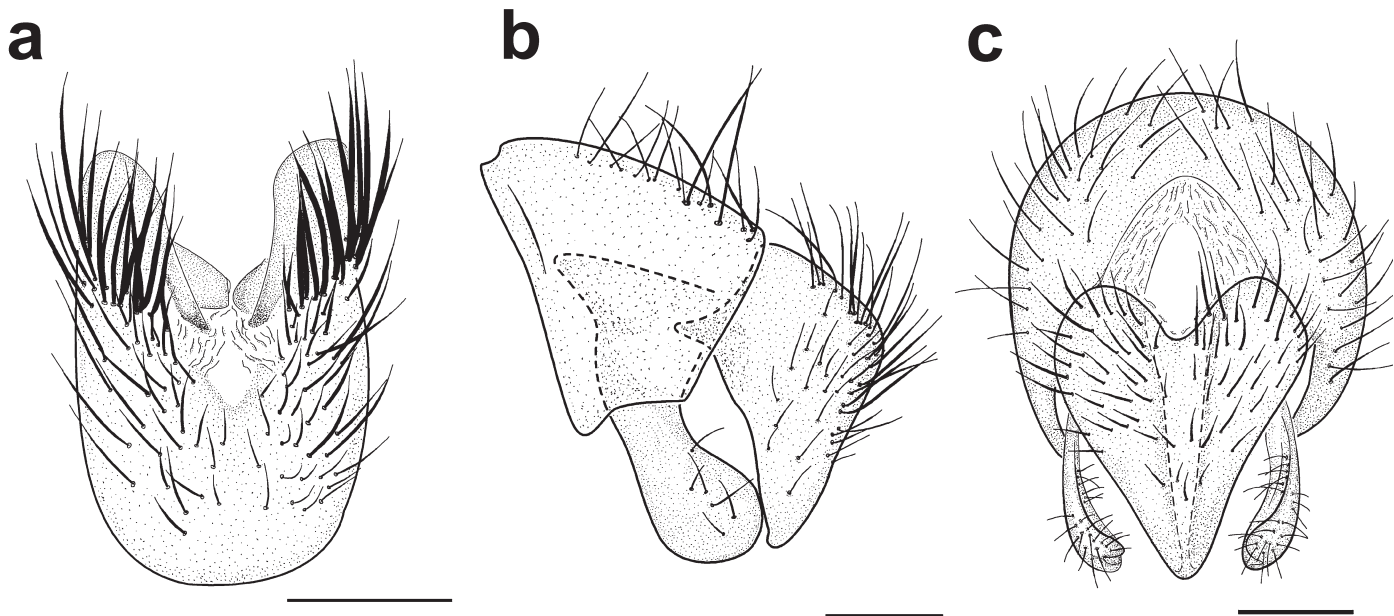

Fig. 3. Terminalia of male Helina fratercula (Zetterstedt, 1845). - a. Sternite 5 , ventral view. $-b$. Terminalia, lateral view. - c. Cercus, dorsal view. Scale bars $0.5 \mathrm{~mm}$.

spine conspicuous, about as long as crossvein $\mathrm{r}$ $m$, vein $S c$ sinuate, six ventral setae at node of Rs, vein $R_{4+5}$ and $M$ slightly diverging from each other in distal part, crossvein r-m with cloudy appearance, crossvein dm-cu straight (Fig. 2a); calypters yellowish, lower one projecting beyond upper one, haltere brownish-yellow. Legs. Tibiae and apex of mid and hind femora yellow, remaining parts black; fore tibia with 1 median $p$ and 1 distal $d$; mid coxa with strong setae on lower and inner margins (Fig. 2d), mid femur with a row of $p v$, strong in basal half, fine towards apex, mid tibia with a row of $p d$ (about 6); hind femur with complete $a d$ row and a row of $a v$ (distal 1/2), hind tibia with 1 row of $a v$ (about 12-15), 1 row of $a d$ and 3 slender $p d$ (in basal half).

Abdomen. Conical, ground-colour black, with densely yellowish gray pollinosity, without distinct median vitta and lateral marks; abdominal tergites 2-4 each with a pair of small spots; sternite 1 without any seta, sternites 2-4 with sparse setae, and with elongated setae on posterior margins. Sternite 5 with strong and dense setae along basal part of each processes (Fig. 3a). Terminalia: Cercus with a broad base and heartshaped in posterior view (Fig. 3c). Surstylus expanded in distal part and with a rounded tip (Fig. $3 b$ ), and with numerous fine setae on distal part (Fig. 3c).

Distribution. China (new record), Austria, Czech Republic, Germany, Poland, Slovakia, Switzerland.
Remarks. Only two specimens of $H$. fratercula were obtained from Xinjiang Uygur Autonomous Region in the Altai Mountains of China. This is the first record of the species in China and the first record out of Central Europe. The species has appeared in the literature without any detailed photos and illustrations (e.g. Zetterstedt 1845, Hennig 1955-1964). However, the characteristic photos and the illustrations of male terminalia are very important for species identification and phylogenetic studies. Helina fratercula can be distinguished from other Helina species by katepisternal setae 1:2, with strong setae on lower margin (Fig. 2d); node of Rs with ventral setae (Fig. 2c); and mid coxa with strong setae on lower and inner margins (Fig. 2d). Helina fratercula can be incorporated into the key of Helina males of China (Xue et al. 2005) as follows:

7 Scutellum bare on ventral surface; pra hairlike or slightly longer than hairs

8

- Scutellum with hairs on ventral surface; pra about $1 / 2$ times as long as the second notopleural seta

9

8 Katepisternal setae 2:2; presutural acr in four rows, postsutural $d c$ usually 4 ; frontal vitta about as wide as anterior ocellus, parafacial as wide as antennal flagellomere, the longest hair of arista as long as width of flagellomere H. confinis (Fallén)

- Katepisternal setae 1:2 (Fig. 2d) 8a, b 
8a Arista long plumose, the longest hair about 2.0 times as width of antennal flagellomere; vein $\mathrm{M}$ distinctly curved toward $\mathrm{R}_{4+5}$ in distal part; hind tibia with 1 ad

H. magnimaculata Feng [subjective junior synonym of Myospila lenticaps (Thomson), as revised by Wang (2013)]

$8 \mathrm{~b}$ Arista short plumose, the longest hair shorter than $1 / 2$ width of antennal flagellomere; vein $\mathrm{R}_{4+5}$ and $\mathrm{M}$ slightly diverging from each other in distal part (Fig. 2a), hind tibia with 1 row of ad (about 12-15) H. fratercula (Zetterstedt)

Acknowledgements. This study was supported by the Beijing Higher Education Young Elite Teacher Project (No. YETP0771), Program for New Century Excellent Talents in University (No. NCET-12-0783), and National Science Foundation of China (No. 31201741, 31272347).

\section{References}

Carvalho, C. J. B. de, Couri, M. S., Pont, A. C., Pamplona, D. \& Lopes, S. M. 2005: A Catalogue of the Muscidae (Diptera) of the Neotropical Region. - Zootaxa 860: 1-282.

Hennig, W. 1955-1964: Muscidae. - In: Lindner, E. (ed.), Die Fliegen der palaearktischen Region. 63b. E. Schweizerbart, Stuttgart. 1110 pp.

McAlpine, J. F. 1981: Morphology and terminology adults. - In: McAlpine, J. F., Peterson, B. V., Shewell,
G. E., Teskey, H. J., Vockeroth, J. R. \& Wood, D. M. (eds.), Manual of Nearctic Diptera, 1. Research Branch, Agriculture Canada Monograph 27: 9-63. Canadian Government Publishing Center, Hull. 674 pp.

Pont, A. C. 1980: Family Muscidae. — In: Crosskey, R. W. (ed.), Catalogue of the Diptera of the Afrotropical Region: 721-761. British Museum (Natural History), London. 1437 pp.

Pont, A. C. 1986: Family Muscidae. — In: Soós, Á. \& Papp, L. (eds.), Catalogue of Palaearctic Diptera. Volume 11, Scathophagidae-Hypodermatidae: 57-215. Akadémiai Kiadó, Budapest. 346 pp.

Stuckenberg, B. R. 1999: Antennal evolution in the Brachycera (Diptera), with a reassessment of terminology relating to the flagellum. - Studia dipterologica 6 : 33-48.

Wang, M. F. 2013: New synonymies and new combinations of Muscidae from China (Diptera, Muscoidea). - ZooKeys 290: 31-38.

Wang, M. F., Zhang, D. \& Wang, R. R. 2008: Two new species of the genus Helina Robineau-Desvoidy (Diptera: Muscidae) from China with revised diagnosis on the Chinese species. - Annales de la Société entomologique de France 44: 139-144.

Xue, W. Q. \& Chao, C. M. 1998: Flies of China. - Liaoning Science and Technology Press, Shenyang. 1365 pp. [In Chinese.]

Xue, W. Q., Feng, Y. \& Tong, Y. F. 2005: A taxonomic study on Helina R.-D. (Diptera: Muscidae) from China. — Oriental Insects 39: 35-62.

Zetterstedt, J. W. 1845: Diptera Scandinaviae. Disposita et descripta. Tomus quartus. - Officina Lundbergiana, Lundae: 1281-1738. 